\title{
CanvasEmb: Learning Layout Representation with Large-scale Pre-training for Graphic Design
}

\author{
Yuxi Xie ${ }^{1 *}$, Danqing Huang ${ }^{2}$, Jinpeng Wang ${ }^{3 *}$, Chin-Yew Lin ${ }^{2}$ \\ ${ }^{1}$ National University of Singapore, ${ }^{2}$ Microsoft Research Asia, ${ }^{3}$ Meituan \\ xieyuxi@u.nus.edu,dahua@microsoft.com,wjp.pku@gmail.com,cyl@microsoft.com
}

\begin{abstract}
Layout representation, which models visual elements and their inter-relations in a canvas, plays a crucial role in graphic design intelligence. With a large variety of layout designs and the unique characteristic of layouts that visual elements are defined as a list of categorical (e.g., type) and numerical (e.g., position and size) properties, it is challenging to learn general and compact representations with limited data. Inspired by the recent success of self-supervised pre-training techniques in various natural language processing tasks, in this paper, we propose CanvasEmb (Canvas Embedding), which pre-trains deep representations from unlabeled graphic designs by jointly conditioning on all the context elements in a canvas, with a multi-dimensional feature encoder and a multi-task learning objective. The pre-trained CanvasEmb model can be fine-tuned with just one additional output layer and with a small size of training data to create models for a wide range of downstream tasks. We verify our approach with presentation slides data. We construct a large-scale dataset with more than one million slides, and propose two layout understanding tasks with human-labeled sets, namely element role labeling and image captioning. Evaluation results on these two tasks show that our model with fine-tuning achieves state-of-the-art performance. Furthermore, we conduct a deep analysis aiming to understand the modeling mechanism of CanvasEmb, and demonstrate its great potential with two extended applications: layout auto completion and layout retrieval.
\end{abstract}

\section{CCS CONCEPTS}

\section{- Information systems $\rightarrow$ Multimedia content creation.}

\section{KEYWORDS}

neural networks, pre-training, layout representation, graphic design

\section{ACM Reference Format:}

Yuxi Xie ${ }^{1 *}$, Danqing Huang ${ }^{2}$, Jinpeng Wang ${ }^{3 *}$, Chin-Yew Lin ${ }^{2}$. 2021. CanvasEmb: Learning Layout Representation with Large-scale Pre-training for Graphic Design. In Proceedings of the 29th ACM International Conference on Multimedia (MM '21), October 20-24, 2021, Virtual Event, China. ACM, New York, NY, USA, 9 pages. https://doi.org/10.1145/3474085.3475541

\footnotetext{
${ }^{*}$ This work was done when the first author was an intern and the third author was an employee at Microsoft Research Asia.

Permission to make digital or hard copies of all or part of this work for personal or classroom use is granted without fee provided that copies are not made or distributed for profit or commercial advantage and that copies bear this notice and the full citation on the first page. Copyrights for components of this work owned by others than the author(s) must be honored. Abstracting with credit is permitted. To copy otherwise, or republish, to post on servers or to redistribute to lists, requires prior specific permission and/or a fee. Request permissions from permissions@acm.org.

MM '21, October 20-24, 2021, Virtual Event, China

(c) 2021 Copyright held by the owner/author(s). Publication rights licensed to ACM. ACM ISBN 978-1-4503-8651-7/21/10 ..\$15.00

https://doi.org/10.1145/3474085.3475541
}

\section{INTRODUCTION}

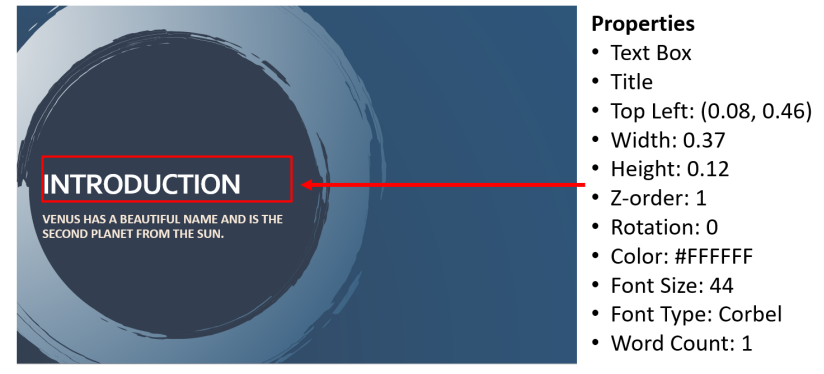

Figure 1: An example layout for a slide. Each element contains multiple properties, making it complex and diverse.

Graphic design layout is referred to the way in which we arrange visual elements in a canvas, as to present information in a logical and coherent way for many different types of documents. Layout representation is the process of modeling the visual elements and their inter-relations, which is the key for analysis [25], retrieval [1] and generation $[10,13]$. Learning general and compact representations for layouts would potentially benefit downstream tasks of graphic designs. However, this task is very challenging due to the rich diversity of layouts and the limited amount of data. Layouts can be very complex and diverse, since a layout contains structured and hierarchical information, from the relations between multiple elements to each individual element which is defined with multidimensional properties such as type (e.g., text box, image or button), position and color. As illustrated in the presentation slide shown in Figure 1, a slight adjustment on the elements would make it a different layout.

Previous works related to layout representation [10, 11, 27] are mostly task-oriented. They simplify the layout only as types and positions of elements, and directly optimize task-specific labels with less than a few thousands instances. Recently a majority of selfsupervised pre-trained models such as ELMO [19], GPT [20] and BERT [3] have shown promising results in improving a variety of natural language processing (NLP) tasks, as well as other domains such as programming codes [5], scanned word documents [32] and videos [26]. The success of pre-trained models has inspired us to learn contextual layout representations from large-scale unlabeled graphic designs, and testify its effectiveness in applications of design intelligence.

In this paper, we present CanvasEmb, a large-scale pre-trained model for learning contextual layout representation. It is designed to be pre-trained to learn deep representations from unlabeled graphic designs by jointly conditioning on all the context elements in a canvas, which can be further fine-tuned with just one additional output layer and on a small size of training data for a wide range 
of downstream tasks. Specifically, we define a generic and comprehensive vocabulary to describe element properties in the canvas. A feature encoder is designed to jointly learn multi-dimensional properties, which is further developed with the multi-layer Transformer [3] to extract the contextualized information of elements. To enhance the contextualization in the representations of elements, we adopt the masked language modeling strategy for multiple properties via multi-task training, where we randomly and selectively mask some of the properties of some of the elements for regeneration in the pre-training.

To verify our approach, we construct a large-scale dataset with more than one million presentation slides, which contains rich layout meta-information for pre-training. Motivated by applications in practice, we propose two downstream tasks of layout understanding to evaluate the performance of our pre-trained CanvasEmb model. The first task is element role labeling, aimed to classify the role of each element (e.g., heading, decorator) given a set of application-specific role labels. The second task is image captioning, which is to detect if a text box and an image in a slide are connected with the image captioning relation. Experimental results on the two tasks show that fine-tuning the CanvasEmb model achieves stateof-the-art performance. Furthermore, we conduct a deep analysis to understand the intrinsic modeling mechanism of CanvasEmb. Also, we demonstrate the great potential of our pre-trained CanvasEmb to be applied on two extended tasks of layout auto completion [13] and layout retrieval.

The contributions of this work are as follows:

- We propose CanvasEmb, which to the best of our knowledge is the first pre-trained model for layouts in graphic design. CanvasEmb can be fine-tuned with a small size of training data to be applied on a wide range of downstream tasks.

- We construct a large-scale dataset of presentation slides with rich layout information, as well as two tasks for layout understanding (i.e., element role labeling and image captioning) with human-labeled sets.

- We show that our model achieves state-of-the-art performance on both two downstream tasks, and further demonstrate its potential for extended applications via the tasks of layout auto completion and layout retrieval respectively.

\section{RELATED WORK}

In this section, we review previous works related to layout representation and pre-trained models.

\subsection{Layout Representation and Applications}

Layout is composed with visual elements which are generally defined by properties such as type and position. Traditional approaches $[1,2,4,25]$ use hand-crafted features to represent a layout, specific for each different application. For example, [25] designs features related to position, spacing and font styles for document structure analysis. More recent approaches [11, 18, 24, 34] encode layouts using neural networks (e.g., tree-LSTM [28], Transformer [29], Faster-RCNN [22]) to obtain the low-dimensional continuous representations, which shows promising results. Training in most previous works are supervised with task-specific labels, or self-supervised such as LayoutVAE [7] and LayoutGAN [12] which are proposed for scene layout generation. In this paper, we adapt the Transformer-based architecture and aim to pre-train a deep representation for layouts with large-scale unlabeled data.

There are increasing applications of graphic design intelligence. Element role detection for document structure analysis [24, 25] identifies the roles of the elements in a layout. Layout auto completion $[10,13]$ aims to predicts the bounding boxes of remaining elements given the input of an empty or partial layout. Other tasks, such as layout generation [7, 10, 12, 18, 27] and layout evaluation [15], are also interesting topics. This paper adopts several applications including element role detection and layout auto completion mentioned above for evaluation, as to verify if the pre-trained representation would enable its great power in the area of graphic design intelligence.

\subsection{Pre-trained Models}

Pre-trained models have shown to bring great improvements to downstream tasks in computer vision $(\mathrm{CV})[6,23]$, natural language processing (NLP) $[3,19,20,33]$ and other domains (e.g., programming codes [5], videos [26]). The problem of learning general representations for graphic design layouts has been rarely studied. The most related work recently, LayoutLM [32], works on scanned document images and jointly models text and layout (defined only as the bounding box of each word token). Different from them, we consider a more general definition of layout, and focus on layout part only as layout itself has its own uniqueness and challenges that remain unexplored. In the next sections, we will show our modeling approach and experiments with detailed analysis.

\section{LAYOUT IN GRAPHIC DESIGN}

Layout in graphic design refers to the way in which we arrange the visual elements on a canvas. Though some settings might vary specific to different document types (e.g., presentation slides, web pages, posters), there exists basic characteristics of elements that make up the content of layouts (example shown in Figure 1):

- Type Properties. Elements can be text boxes, pictures or lines. In different scenarios there can be different semantic roles. For example, elements can be divided into title, subtitle or other functional labels in presentation slides.

- Geometry Properties. Position and size indicate the elements' placement in the layout. Besides, z-order is the ordering of overlapping two-dimensional elements, and rotation describes an element's circular movement.

- Color Properties. Color is one of the most straightforward visual feature, including the RGBA channels and extra features such as color gradient.

- Content-related Properties. Though user contents are separated from the layout, some related properties such as fonts and text length can affect the element arrangement.

As elements are complex and sparse with properties of either categorical (e.g., type, color) or numerical (e.g., position, font size) values, layouts are diverse and complicated for modeling. In the next section, we will introduce our approach for layout representation learning. 


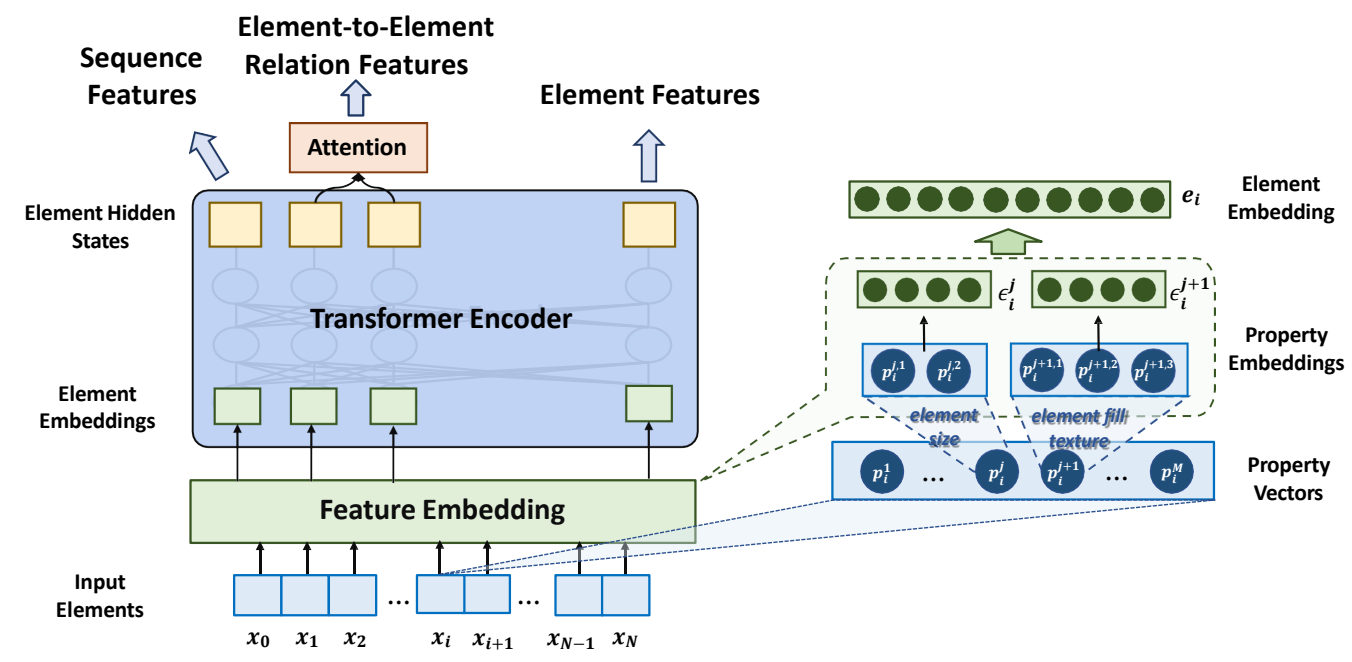

Figure 2: Overall Architecture of CanvasEmb (left) with the details of feature embedding (right). The three levels of features can be extracted on top of the Transformer Encoder outputs for fine-tuning on various downstream tasks. Here the attention mechanism is an additional part for the fine-tuning task about element relations.

\section{MODELING}

We present our model CanvasEmb, which inputs elements with multi-dimensional properties and outputs representation for the layout. To train our model, we adopt the two-stage learning framework, namely pre-training and fine-tuning.

\subsection{Model Architecture}

We formulate the input as a sequence of visual elements $\left\{\mathbf{x}_{i}\right\}_{i=0}^{n}$ in the layout, where each element $\mathbf{x}_{i}$ is defined with $m$ properties $\left\{\mathbf{p}_{i}^{j}\right\}_{j=1}^{m}$. Here $\mathbf{x}_{0}$ is the sequence representation which is randomly initialized. Figure 2 shows the overview architecture of our model, which is similar to BERT [3]. The feature embedding encodes high dimensional properties for elements, and is concatenated with the Transformer encoder to model the global context of elements. The output representation can be further used to make prediction of element-level and layout-level labels, as well as the relations between the elements, with an extra task-specific prediction layer. Here, we introduce the details of model components.

4.1.1 Feature Embedding. For the $i$-th element $\mathbf{x}_{i}=\left[\mathbf{p}_{i}^{1} ; \mathbf{p}_{i}^{2} ; \ldots ; \mathbf{p}_{i}^{m}\right]$, the embedding $\mathbf{e}_{i}$ is obtained by concatenating $m$ property embeddings $\left\{\epsilon_{i}^{j}\right\}_{j=1}^{m}$ :

$$
\mathbf{e}_{i}=\Theta\left(\epsilon_{i}^{1} \oplus \epsilon_{i}^{2} \oplus \ldots \oplus \epsilon_{i}^{m}\right),
$$

where $\oplus$ is the concatenation operator and $\Theta$ is a non-linear transform function.

For each channel $j$, the corresponding property $\mathbf{p}_{i}^{j}$ contains multi-dimensional values. For example, given a 2-dim numerical property $\mathbf{p}_{i}^{j}=\left[p_{i}^{j, 1} ; p_{i}^{j, 2}\right]$ (e.g., element size with height and width), the embedding in this channel can be calculated as:

$$
\epsilon_{i}^{j}=\xi^{j}\left(p_{i}^{j, 1} \oplus p_{i}^{j, 2}\right),
$$

where $p_{i}^{j, k}$ represents the $k$-th value in $\mathbf{p}_{i}^{j}$ and $\xi^{j}$ is the embedding function.

There are two types of embedding functions. For properties with categorical values such as type and color, we use the embedding matrix as the learning parameter. For properties with numerical values such as position and size, the positional encoding [29] is adopted:

$$
\begin{gathered}
\left.P E_{(} p_{i}^{j, k}, 2 h\right)=\sin \left(p_{i}^{j, k} / 10000^{2 h / d^{j, k}}\right) \\
\left.P E_{(} p_{i}^{j, k}, 2 h+1\right)=\cos \left(p_{i}^{j, k} / 10000^{2 h / d^{j, k}}\right)
\end{gathered}
$$

where $d^{j, k}$ means the embedding dimension assigned to $p_{i}^{j, k}$ and $h$ is the dimension.

4.1.2 Transformer Encoder. On top of the feature embeddings, we use a Transformer [29] to encode the element contexts. Similar to BERT [3], the multi-layer Transformer with the multi-head selfattention mechanism enables to capture correlations between different elements and property fields. Specifically, we adopt the querykey-value attention mechanism in Transformer. Taking the $i$-th element $\mathbf{h}_{i}$ and $j$-th element $\mathbf{h}_{j}$ as an example, the attention score of the $h$-th head can be calculated as:

$$
\begin{gathered}
\alpha_{i, j}^{(h)}=\frac{\exp \phi^{(h)}\left(\mathbf{h}_{i}, \mathbf{h}_{j}\right)}{\sum_{t=0}^{n} \exp \phi^{(h)}\left(\mathbf{h}_{i}, \mathbf{h}_{t}\right)}, \\
\phi^{(h)}\left(\mathbf{h}_{i}, \mathbf{h}_{j}\right)=\frac{\mathbf{W}_{Q}^{(h)} \mathbf{h}_{i} \cdot \mathbf{W}_{K}^{(h)} \mathbf{h}_{j}}{\sqrt{d_{K}}}
\end{gathered}
$$

where $\phi^{(h)}$ means the attention function for the $h$-th head, and $d_{K}$ is the dimension of the key vectors.

$$
\begin{gathered}
\mathbf{h}_{i}^{(h)}=\sum_{j=0}^{n} \alpha_{i, j}^{(h)} \mathbf{W}_{V}^{(h)} \mathbf{h}_{j} \\
\mathbf{h}_{i}^{\prime}=\mathbf{W}_{O}\left[\mathbf{h}_{i}^{\prime(1)} \oplus \mathbf{h}_{i}^{\prime(2)} \oplus \ldots \oplus \mathbf{h}_{i}^{\prime(H)}\right]
\end{gathered}
$$

Then the hidden states of the elements are updated as above, based on these attention scores and another sequence of weight matrices $\left\{\mathbf{W}_{V}^{(h)}\right\}_{h=1}^{H}$, where $H$ is the number of heads. The updated hidden state in each head $\mathbf{h}_{i}^{\prime(h)}$ are then aggregated together by a weight matrix $\mathbf{W}_{O}$ for the $i$-th element. Finally, we can get the 
low-dimensional representations $\left\{\mathbf{h}_{i}^{(l)}\right\}_{i=0}^{n}$ for all elements from the last, i.e. the $l$-th encoding layer:

$$
\left(\mathbf{h}_{0}^{(l)}, \mathbf{h}_{1}^{(l)}, \ldots, \mathbf{h}_{n}^{(l)}\right)=\left\{\begin{array}{lr}
\mathcal{T}_{l}\left(\mathbf{e}_{0}, \mathbf{e}_{1}, \ldots, \mathbf{e}_{n}\right), & l=1 \\
\mathcal{T}_{l}\left(\mathbf{h}_{0}^{(l-1)}, \mathbf{h}_{1}^{(l-1)}, \ldots, \mathbf{h}_{n}^{(l-1)}\right), & 2 \leq l \leq L
\end{array}\right.
$$

where $\mathcal{T}_{l}$ represents the $l$-th layer of the Transformer encoder.

\subsection{Pre-training CanvasEmb}

We adopt the masked language modeling (MLM) as the objective of CanvasEmb pre-training, which has been proven effective in several domains $[3,16,26]$. To enable the model to learn correlations from different properties, we randomly select any one type of properties to mask for an element during training. CanvasEmb is trained to approximately maximize the pseudo likeli$\operatorname{hood} E_{\mathbf{x} \sim \mathcal{D}} \sum_{i=1}^{n} \sum_{j=1}^{m} P\left(\mathbf{p}_{i}^{j} \mid \mathbf{p}_{i}^{\backslash j}, \mathbf{x}_{\backslash i} ; \theta\right)$, where $\mathcal{D}$ is the real data distribution, $\theta$ represents the model parameters, $\mathbf{p}_{i}^{\backslash j}$ represents the properties masking $p_{i}^{j}$ of the $i$-th element, and $\mathbf{x}_{\backslash i}$ means all the elements in the input excluding the $i$-th one. Particularly, we adopt cross-entropy loss and mean-square error loss for classification and regression tasks respectively:

$$
\mathcal{L}(\theta)=\left\{\begin{array}{l}
\sum_{i=1}^{n} \sum_{j \in \mathcal{M}_{c}}-\log P\left(\mathbf{p}_{i}^{j} \mid \mathbf{p}_{i}^{\backslash j}, \mathbf{x}_{\backslash i} ; \theta\right) \\
\sum_{i=1}^{n} \sum_{j \in \mathcal{M}_{u}}\left\|\mathbf{p}_{i}^{j}-\hat{\mathbf{p}}_{i}^{j}\right\|_{2}
\end{array}\right.
$$

Here $\mathcal{M}_{c}$ and $\mathcal{M}_{u}$ represent the index sets of categorical and numerical properties, and $\hat{\mathbf{p}}_{i}^{j}$ is the model prediction.

\subsection{Fine-tuning CanvasEmb}

For different downstream tasks, we feed the same input as in the pre-training phase, and fine-tune the model with extra task-specific layers:

Element-Level tasks are aimed to predict specific features of the elements, e.g. properties which are different from but correlated to the input ones.

Element-to-Element tasks to predict the relations between a pair of elements.We build a query-memory attention-based mechanism for the relation prediction [30], where the relation score is calculated as:

$$
\begin{gathered}
\mathcal{R}\left(\mathbf{x}_{i}, \mathbf{x}_{j}\right)=\alpha_{i, j}+\alpha_{j, i} \\
\alpha_{i, j}=\frac{\exp W_{Q}^{r} \mathbf{h}_{i}^{(L)} \cdot W_{M}^{r} \mathbf{h}_{j}^{(L)}}{\sum_{j^{\prime}=0}^{n} \exp W_{Q}^{r} \mathbf{h}_{i}^{(L)} \cdot W_{M}^{r} \mathbf{h}_{j^{\prime}}^{(L)}}
\end{gathered}
$$

where $W_{Q}^{r}$ and $W_{M}^{r}$ are trainable transforming weight matrices for a specific task $r$.

Sequence-Level tasks utilize the sequence representation token $\mathbf{x}_{0}$ to make layout-wise prediction.

\section{EXPERIMENT}

In this section, we perform experiments on a new presentation slide dataset, and conduct in-depth analysis on the model performance on the downstream tasks and extended applications.

\subsection{Dataset and Tasks}

CanvasEmb is designed to represent various types of graphic designs. In this paper, we adopt presentation slides to construct our layout design dataset, as a huge amount of slides are publicly available and the understanding of slides would enable a wide range of applications in practice. Given a slide, we extract the properties of each element using a slide parsing tool ${ }^{1}$. Each slide is represented as a set of elements with multiple properties, including type, place-

\begin{tabular}{|c|c|}
\hline Property & Description \\
\hline Type & $\begin{array}{l}\text { Type of an element (e.g., text-box, image, } \\
\text { chart, geometric shapes). }\end{array}$ \\
\hline Placeholder & $\begin{array}{l}\text { Default semantic role of an element (e.g., ti- } \\
\text { tle, subtitle, bodytext). This label can be very } \\
\text { noisy, as user might create a title by insert- } \\
\text { ing a new text-box which will be labeled as } \\
\text { bodytext by default. }\end{array}$ \\
\hline Position & Normalized bounding box of an element. \\
\hline Size & Normalized width and height of an element. \\
\hline Z-Order & $\begin{array}{l}\text { Z-Order of an element, indicating whether } \\
\text { one element can cover another when they } \\
\text { overlap with each other. }\end{array}$ \\
\hline Rotation & Rotation angle of an element from $0^{\circ}$ to $360^{\circ}$. \\
\hline Color & $\begin{array}{l}\text { RGBA Value of the fill color of an element, } \\
\text { including color gradient, solid, and pattern. }\end{array}$ \\
\hline Font & $\begin{array}{l}\text { Font type and font size of the text in an ele- } \\
\text { ment. }\end{array}$ \\
\hline Text Statistics & $\begin{array}{l}\text { If an element contains text, consider its } \\
\text { char/word/line count and other text style fea- } \\
\text { tures such as whether the text contains url. }\end{array}$ \\
\hline
\end{tabular}
holder, position, size, z-order, rotation, color, font and text statistics. The properties with descriptions are listed in Table 1.

Table 1: Properties of an element used in CanvasEmb.

Pre-training Dataset. We crawl presentation slides from Microsoft Power Point at run-time and collect a pre-training dataset constructed of one million pages of slides. The average number of elements per slide is 2.86. Considering user privacy, we also conduct data cleaning to remove the privacy-sensitive (e.g., detailed contents in text-boxes or user-defined images) and non-layout-related information.

The design of our two evaluation tasks is motivated by the practical application of slide layout transformation, which maps elements in the user slides to placeholders in the well-designed slide templates. To ensure the appropriate mapping, we first need to detect in the user slide (1) the semantic role of each element; (2) elements that belong to the same group (e.g., image captioning). The two tasks are described as follows:

\footnotetext{
${ }^{1}$ We use an internal slide parsing tool similar to the open-source library https://pypi.org/project/python-pptx/.
} 
Downstream task 1: Element Role Labeling. To detect the semantic role of each element on a canvas. As shown in Figure 3, each element is assigned with one of the following seven labels ${ }^{2}$ : title, subtitle, footer, decorator, heading, caption, and other. We separately create a dataset containing 48, 309 slides and split it into $38,819 / 9,490$ for training/testing. Each element on the slide is annotated by 5 annotators ${ }^{3}$ and we use majority voting to assign the final labels. The percentages of different roles in the whole labeling set are: title $(21.28 \%)$, subtitle $(1.24 \%)$, footer $(1.44 \%)$, decorator (0.44\%), heading $(0.20 \%)$, caption $(1.23 \%)$ and other $(72.32 \%)$. As we can see, the class distribution sampled from the real user slides are extremely imbalanced with very few training data for some classes, which makes the task more challenging.

Downstream task 2: Image Captioning. Given a pair of an image and a text box in a slide, the goal is to detect if the two elements match with each other as an image captioning pair (e.g., the bottom right pair in Figure 3 ). With a similar annotation pipeline, we finally get 2, 996 labeled slides, each with at least one image captioning pair, divided as 1,496/1, 500 for training/testing. The average number of the pairs per slide is 3.14 .

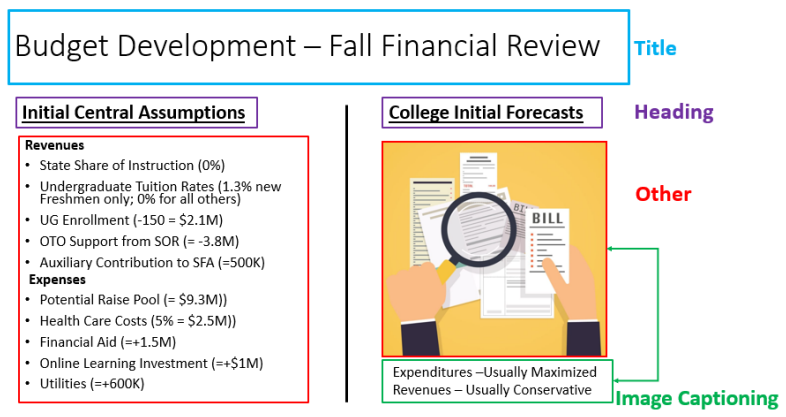

Figure 3: Example slide and annotations for the two evaluation tasks.

\subsection{Set Up}

5.2.1 Model and Training Parameters. Following [3], we testify the effect of the model size by comparing two sizes: CanvasEmb (Base) $(\mathrm{L}=3, \mathrm{H}=64)$ and CanvasEmb (Large) $(\mathrm{L}=6, \mathrm{H}=128)$, where $L$ and $H$ denote the number of encoder layers and the hidden state size respectively.The maximum sequence length during training is 16 for both models. During pre-training, we randomly select one type of property to mask for $30 \%$ elements in each sample by replacing it with $[M A S K]$ ( $90 \%$ of the time) or random values (10\% of the time). For multi-task learning, the loss of each prediction task is weighted according to the current performance on the validation set, where the weight for task $p \in \mathcal{M}_{c}$ is $\log \max \left(\frac{1}{\text { accurac }_{p}+\epsilon}, 1\right)$ and for task $p \in \mathcal{M}_{u}$ is $\log \min \left(\frac{\text { squared_error }_{p}}{10}, 100\right)$. For fine-tuning, we adopt the $\alpha$-balanced focal loss [14] to address the label unbalance. The trade-off parameters $\lambda$ in focal loss is 2 . All models are trained using Adam [9] with mini-batch size 64. The learning rate is initially set

\footnotetext{
${ }^{2}$ The set of roles is highly correlated but different to the input placeholder property, with only three overlapping labels title, subtitle, footer.

${ }^{3}$ The annotators are paid according to the numbers of samples they labeled.
}

to $10^{-5}$ and adaptive learning rate decay is applied. We also adopt early stopping and gradient clipping [17]. It takes approximately 18 hours for pre-training and less than 1 hour for fine-tuning using 4 NVIDIA Tesla P100 GPUs. The inference time is a few minutes. We also convert to the ONNX model which takes less than $40 \mathrm{~ms}$ per batch of size 64 .

\subsubsection{Baselines. We compare our model against two baselines:}

(1) Decision Tree implemented as GBDT [8];

(2) Simple-MLP, as we implement a neural baseline with the same feature embedding module in Section 4 concatenated by a MLP of 3 layers on the top.

The baselines share same inputs of element properties. We do not consider previous layout-related works for comparison, since a considerable amount of works [7, 12, 18, 34] are generative models for layout generation which are not applicable in our tasks, while other works [13, 24] share similar neural model architecture as CanvasEmb (e.g., MLP, Transformer), where our approach mainly differ in the design and use of pre-training.

\subsection{Result Analysis}

Now we show the overall results of the two tasks.

Element Role Labeling. We report the F1 score for each role as well as the overall macro/micro F1 metrics in Table 2. On the one hand, CanvasEmb in all settings outperform traditional Decision Tree and the neural baseline with a large margin on most of the metrics. On the other hand, CanvasEmb (Large) achieves better results than CanvasEmb (Base), and pre-training further boosts the performances.

Image Captioning. We report F1 and AUC scores in Table 3. Similarly, CanvasEmb (Large) with pre-trained achieves the best results. Since this task focuses on the relation between elements, we also train the enhanced baselines (+2D features) where we explicitly incorporate additional handcrafted pairwise features, such as the distance between two elements. We observe that CanvasEmb (Large) outperforms the enhanced baseline with a large margin. This indicates the ability of CanvasEmb to capture the relations between elements in a certain degree, which is much more efficient than the heuristic way of manual feature engineering.

\subsection{Effect of Pre-training}

We assess the effect of pre-training under different settings of the model size and the fine-tuning data size.

Correlation between pre-training and the model size. In general, on both tasks pre-trained CanvasEmb models outperform those without being pre-trained. Specifically, the increase of the model size (the large model) further boosts the performance compared to the base model. For example, in the task of element role labeling, the pre-trained model brings $7.56 \%$ macro F1 gain for CanvasEmb (Large) while only $0.05 \%$ gain for CanvasEmb (Base). This is accordant with our intuition, i.e. larger models tend to capture and embody more knowledge from the pre-training.

Correlation between pre-training and the size of the labeled fine-tuning dataset. We also conduct an ablation study with only $30 \%$ labeled training data for both downstream tasks, as shown in Table 2 and 3 respectively. We observe that pre-training contributes more when labeled data size is smaller. In element role 
Table 2: Results for element role labeling.

\begin{tabular}{l|ccccccc|c|c}
\hline $\mathbf{1 0 0 \%}$ labeled data & title & subtitle & footer & heading & decorator & caption & other & macro & micro \\
\hline Decision Tree & 96.62 & 82.28 & 93.24 & 53.79 & 58.45 & 50.34 & 73.59 & 72.62 & 89.34 \\
simple-MLP & 96.73 & 84.16 & 94.64 & 54.81 & 60.01 & 50.18 & 72.15 & 73.24 & 89.86 \\
CanvasEmb (Base) & 95.65 & $\mathbf{9 1 . 7 6}$ & $\mathbf{9 5 . 9 4}$ & 34.55 & 96.92 & 71.15 & 71.48 & 79.64 & 91.77 \\
- w/o pretrained & 95.88 & 90.62 & 95.76 & 39.79 & 97.44 & 64.91 & 72.73 & 79.59 & 91.99 \\
CanvasEmb (Large) & $\mathbf{9 8 . 7 4}$ & 88.28 & 94.18 & $\mathbf{5 5 . 1 5}$ & $\mathbf{9 8 . 6 6}$ & $\mathbf{7 8 . 2 5}$ & $\mathbf{7 8 . 7 3}$ & $\mathbf{8 4 . 5 7}$ & $\mathbf{9 5 . 9 7}$ \\
- w/o pretrained & 95.18 & 88.07 & 92.37 & 30.93 & 97.29 & 70.06 & 65.59 & 77.07 & 90.87 \\
\hline 30\% labeled data & & & & & & & & & \\
\hline Decision Tree & 97.06 & 71.44 & 66.67 & 0.00 & 63.03 & 19.75 & $\mathbf{3 6 . 1 4}$ & 50.58 & 86.62 \\
simple-MLP & 97.14 & 74.84 & 70.56 & 10.16 & 62.27 & 18.16 & 30.49 & 51.95 & 87.73 \\
CanvasEmb (Base) & 97.69 & $\mathbf{8 9 . 7 8}$ & 88.20 & 60.56 & 95.40 & 75.80 & 29.18 & 76.66 & 93.16 \\
- w/o pretrained & 93.67 & 86.61 & 82.83 & 48.44 & 95.71 & 58.03 & 19.16 & 69.21 & 86.88 \\
CanvasEmb (Large) & $\mathbf{9 8 . 9 8}$ & 87.46 & 88.20 & $\mathbf{6 1 . 5 9}$ & 95.40 & $\mathbf{7 5 . 8 0}$ & 29.18 & $\mathbf{7 6 . 6 6}$ & $\mathbf{9 5 . 4 2}$ \\
- w/o pretrained & 94.08 & 88.18 & $\mathbf{9 2 . 5 1}$ & 51.73 & $\mathbf{9 5 . 8 9}$ & 73.08 & 15.89 & 73.05 & 88.18 \\
\hline
\end{tabular}
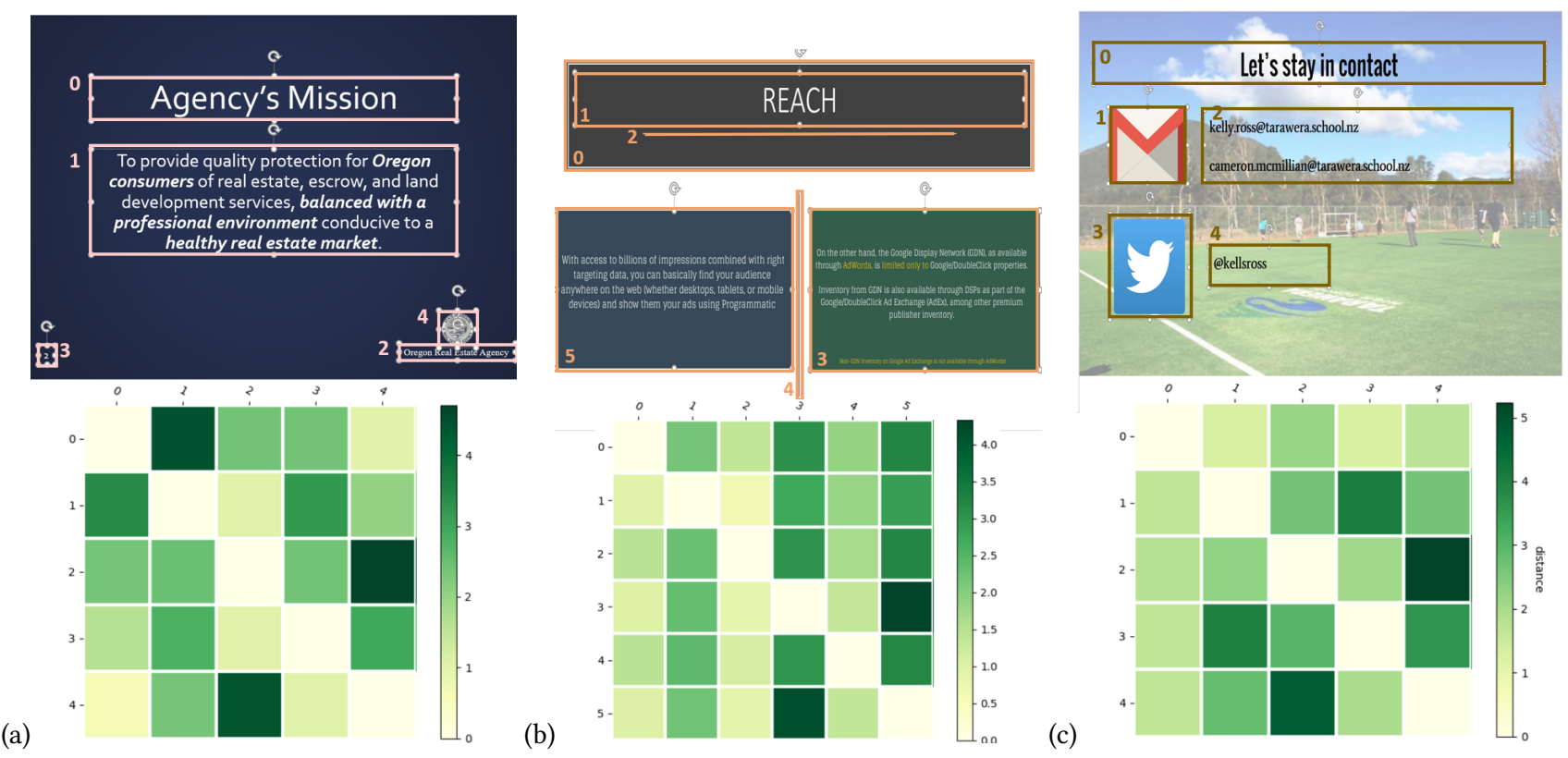

(a)

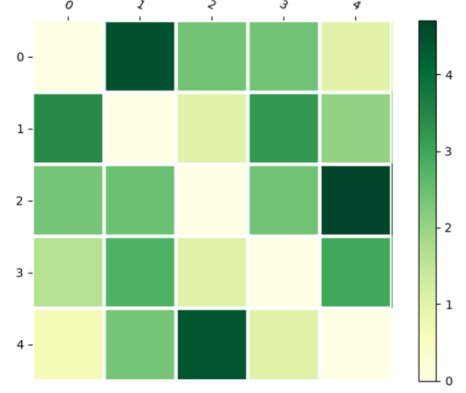

(b) (c)

Figure 4: The element-to-element relations learnt in three different tasks: (a) Pre-training (b) Fine-tuning on the element-rolelabeling task (c) Fine-tuning on the image-captioning task. The first row shows the original slides with element indexes, and the second row shows the corresponding heatmaps where $\mathrm{X}$ - and $\mathrm{Y}$-axis are the element indexes.

labeling task, there is $\Delta=7.45 \%$ macro $\mathrm{F} 1$ gain from pre-training for CanvasEmb (Base) under the setting of $30 \%$ training data, compared to only $0.05 \%$ gain using the full data. Especially for those minority classes (e.g., heading, caption) with few training instances, the improvement from pre-training is more significant. For example, the F1 score of heading increases from 51.73 to 61.59 with pre-training CanvasEmb (Large). Similarly, in the task of image captioning, there is $\Delta=2.68 \% \mathrm{~F} 1$ increase for CanvasEmb (Base) from pre-training using $30 \%$ data while pre-training makes fairly no contribution using the full data. This shows that our pre-trained CanvasEmb can provide a stable initialization which alleviates the data scarcity issue in downstream tasks.

\subsection{Probing into Shape Context}

To investigate how CanvasEmb captures element-to-element relations, we use the impact function [31]

$$
f_{\text {impact }}\left(\mathbf{x}_{i}, \mathbf{x}_{j}\right)=\operatorname{dist}\left(\mathbf{h}_{i}\left(\mathbf{X} \backslash\left\{\mathbf{x}_{i}\right\}\right), \mathbf{h}_{i}\left(\mathbf{X} \backslash\left\{\mathbf{x}_{i}, \mathbf{x}_{j}\right\}\right)\right)
$$

to show the importance of the elements by calculating the distance between the hidden states of the layout when removing elements 
Table 3: Results for image captioning

\begin{tabular}{l|cc}
\hline 100\% labeled data & F1 & AUC \\
\hline Decision Tree & 84.30 & 98.32 \\
+ 2D features & 84.50 & 98.21 \\
simple-MLP & 85.16 & 98.29 \\
+ 2D features & 86.26 & 98.34 \\
CanvasEmb (Base) & 88.99 & 98.23 \\
- w/o pretrained & 89.28 & 98.33 \\
CanvasEmb (Large) & $\mathbf{9 0 . 9 5}$ & $\mathbf{9 8 . 5 1}$ \\
- w/o pretrained & 89.68 & 98.32 \\
\hline 30\% labeled data & & \\
\hline Decision Tree & 76.39 & 96.42 \\
+ 2D features & $\mathbf{8 1 . 3 4}$ & $\mathbf{9 7 . 3 6}$ \\
simple-MLP & 77.14 & 93.37 \\
+ 2D features & 79.42 & 94.54 \\
CanvasEmb (Base) & 78.37 & 94.26 \\
- w/o pretrained & 75.69 & 93.69 \\
CanvasEmb (Large) & 80.64 & 95.27 \\
- w/o pretrained & 79.43 & 94.85 \\
\hline
\end{tabular}

$\left\{\mathbf{x}_{i}\right\}$ and $\left\{\mathbf{x}_{i}, \mathbf{x}_{j}\right\}$. We sample some representative slides with the corresponding heatmaps which are displayed in Figure 4.

Pre-Training. From Figure 4(a), we observe that elements in close region tend to have stronger influences on each other (shown by darker-colored pixels). For example, element 2 and 4 on the bottom right have significantly strong impacts on each other, but weaker impacts on element 3 on the left. The same pattern applies on element 0 and 1 . This indicates that the pre-trained model can typically capture location information in layout representation.

Element Role Labeling. Figure 4(b) visualizes the model finetuned on the task of element role labeling. We can see that elements $1,3,5$ generally have the most significant impacts on all other elements in the slide. Specifically, element 3 and 5, which are symmetric and have the same semantic roles, strongly affect each other. This indicates that the model might learn representations mainly based on the location information as well as size and semantic roles in the slide.

Image Captioning. For the example in Figure 4(c), the finetuned model pays more attention to images and the corresponding caption-like elements. Element pairs $\{1,3\}$ and $\{2,4\}$ have very strong impacts on each other, while element 0 has the weakest influences in the slide. This is an interesting observation to see how caption pairs in a slide interact with each other, as the model in this case obviously separates the element 0 from others with captioning relation.

\subsection{Error Analysis}

Here we study the limitations of our model by conducting error analysis under the two downstream tasks for future improvement. Here we conclude two main error types as listed below:

(1) Not well capturing relative information between elements. As an example shown in Figure 5(a), the two highlighted text boxes in red are falsely predicted as footer (mostly likely due to their positions in the bottom and small font size). This type of errors can be resolved if the model can better model the relations between elements as there exists two groups of shapes (each contains a chart with two text boxes) with strong relative patterns.

(2) Semantic contents such as text and image can be important. For the task of image captioning, we observe there are a certain amount of error cases that the content of images and text boxes plays an important role. For example in Figure 5(b), our model predicts with high confidence that the highlighted text box (with content "Fennec Fox") is caption of both images. However, if the semantic contents of elements are taken into consideration, the ambiguity would be resolved. In this paper, we currently only focus on modeling the layout part. Multi-modality of layout and content could be a promising future direction.

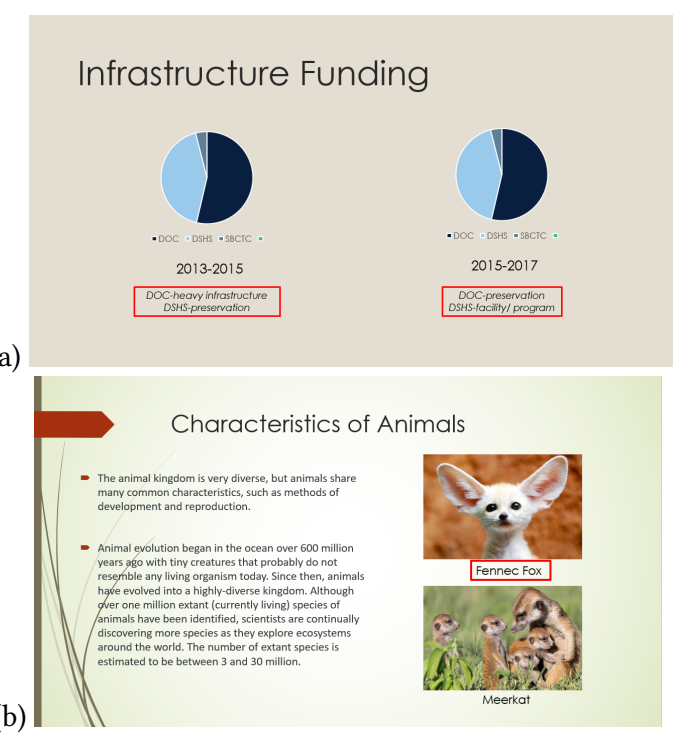

Figure 5: Examples of two main error types. (a) relative information is important: our model predicts the text box in red wrongly as footer where the slide exists two groups of elements with strong relative patterns; (b) modality of semantic contents is important: our model predicts the text box in red as caption for both two images where the content of text and image can resolve the ambiguity.

\section{EXTENDED APPLICATIONS OF PRE-TRAINED CANVASEMB}

In this section, we show more potential uses of our pre-trained models and verify the model effectiveness via two extended applications.

Layout Auto Completion. To further demonstrate the knowledge embodied in our pre-trained CanvasEmb, we show the application of layout auto completion proposed by [13]. Given a partial layout, the model needs to complete the layout by predicting the remaining elements with their properties (e.g., type, position). This task is well-aligned to our pre-training objectives and we can 


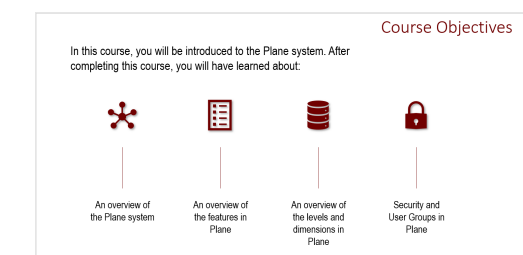

(a)

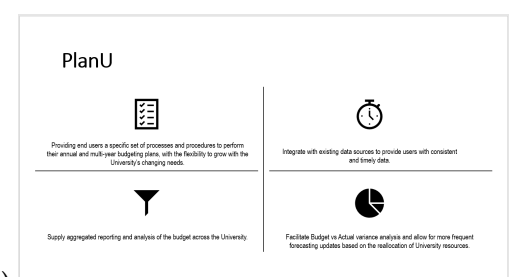

(b)

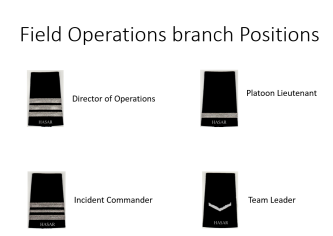

(c)

Figure 6: (a) A query layout and (b)(c) the similar candidates retrieved by CanvasEmb (Large).

directly use our pre-trained models for prediction. We compare with [13] ${ }^{4}$, which also adopts Transformer as the backbone model and differs from ours mainly in its auto-regressive training and task-specific learning objective. Its parameter size is set to the same scale as CanvasEmb (Base). We separately reserve 50,000 slides (each with at least 6 elements) for validation and show the results in Table 4, given different percentages of partial elements in a layout as input, including $10 \%, 50 \%$ and $80 \%$. As we can see, CanvasEmb achieves better results for both type and position, which demonstrates the accurate modeling of elements and effective contextual representations in the pre-trained CanvasEmb.

Table 4: Results for layout auto completion. We show accuracy for Type (T.) IoU for Position (P.)

\begin{tabular}{l|cc|cc|cc}
\hline & \multicolumn{2}{|c}{$10 \%$} & \multicolumn{2}{c}{$50 \%$} & \multicolumn{2}{c}{$80 \%$} \\
\hline Model & T. & P. & T. & P. & T. & P. \\
\hline$[13]$ & 9.16 & 8.87 & 45.28 & 30.77 & 86.03 & 51.97 \\
CanvasEmb (Base) & 9.76 & 10.24 & 46.56 & 32.14 & 86.49 & 52.71 \\
CanvasEmb (Large) & $\mathbf{1 0 . 1 4}$ & $\mathbf{1 1 . 3 6}$ & $\mathbf{4 9 . 8 2}$ & $\mathbf{3 8 . 9 4}$ & $\mathbf{8 8 . 1 7}$ & $\mathbf{5 4 . 1 1}$ \\
\hline
\end{tabular}

Layout Retrieval. Given a query layout, the scenario is to retrieve similar layouts from the database, which can be used for layout adaptation and recommendation. Most previous systems either pre-define layout-related features or input layout as an image with CNN (Convolutional Neural Network) encoders. In this paper, we use CanvasEmb to obtain the representation for each slide and retrieve slides with cosine similarity between the query slide and the candidate slide. For comparison, we train a CNN auto-encoder on the pre-training dataset given the slides in the format of images as a baseline. For evaluation, we randomly sample 100 slides with each containing with at least 5 shapes ${ }^{5}$ from the pre-training dataset as queries, and manually judge the quality of the top 5 retrieved candidate slides from different models. More specifically, three human annotators are invited to rate with scores ranged from 1 to 3 in the following two aspects:

(1) Adaptiveness: the layout of the candidate can be served as a template to the query slide, where elements in the query slide can be mapped to the candidate layout.

(2) Variation: the candidate layout is different from the query with respect to some properties such as position and font setting.

\footnotetext{
${ }^{4}$ We re-implement and train the baseline (vanilla setting) using our pre-training dataset Since there is no hierarchical relations of elements in our slide data, we simplify the setting to predict only type and position, and use accuracy and IoU as the evaluation metrics.

${ }^{5}$ We do not consider slides with less than 5 shapes since the layout variation would be limited for testing.
}

From the human evaluation results in Table 5, CanvasEmb (Large) obtains the highest score (2.31) related to adaptiveness, which in some extend shows the meaningful representations learned by CanvasEmb. As for the criteria of variation, three models perform comparably with scores around 1.60 . We argue that it might be the reason that the goal of variation is not specified in the model's learning objective, and it is natural for models to first retrieve the most similar candidates. The Kappa [21] values on all models range from 0.5-0.9, indicating relatively intermediate to excellent agreement among annotators. Furthermore, we show an example case retrieved by CanvasEmb (Large) in Figure 6. As we can see, both the query and the two candidates contain similarly four images with captions with a certain degree of layout variations.

Table 5: Human evaluation results for layout retrieval: the average scores from the three annotators together (ranged from 1 to 3 , higher is better).

\begin{tabular}{l|cc|cc}
\hline Models & \multicolumn{2}{|c}{ Adaptiveness } & \multicolumn{2}{c}{ Variation } \\
\hline Model & score & Kappa & score & Kappa \\
\hline CNN Auto-encoder & 1.98 & 0.80 & 1.58 & 0.69 \\
CanvasEmb (Base) & 2.28 & 0.85 & $\mathbf{1 . 6 5}$ & 0.58 \\
CanvasEmb (Large) & $\mathbf{2 . 3 1}$ & 0.79 & 1.63 & 0.61 \\
\hline
\end{tabular}

\section{CONCLUSION}

In this paper, we present CanvasEmb, a large-scaled pre-trained model for layout representation in graphic design. It encodes elements in a layout with multi-dimensional properties and models the element context with a Transformer-based architecture. To pretrain CanvasEmb, we adopt the masked language modeling with multi-task learning objective. In the two proposed tasks, fine-tuned CanvasEmb achieves state-of-the-art performances. Furthermore, we conduct a deep analysis to prob into the embodied knowledge learned by CanvasEmb, and show its great potential use via the applications of layout auto completion and layout retrieval. As future work, we are going to apply our model to more downstream tasks, and verify the approach on more document types such as web pages or word documents

\section{ACKNOWLEDGMENTS}

We would like to thank the anonymous reviewers for their valuable reviews. We also thank the annotators for labeling the fine-tuning datasets of downstream tasks. 


\section{REFERENCES}

[1] Joost Beusekom, Daniel Keysers, Faisal Shafait, and Thomas Breuel. 2006. Distance measures for layout-based document image retrieval. In Proceedings of the Second International Conference on Document Image Analysis for Libraries.

[2] Niranjan Damera-Venkata, José Bento, and Eamonn O'Brien-Strain. 2011. Probabilistic Document Model for Automated Document Composition. In Proceedings of the 11th ACM Symposium on Document Engineering (Mountain View, California, USA) (DocEng '11). Association for Computing Machinery, New York, NY, USA $3-12$.

[3] Jacob Devlin, Ming-Wei Chang, Kenton Lee, and Kristina Toutanova. 2019. BERT: Pre-training of Deep Bidirectional Transformers for Language Understanding. In Proceedings of the 2019 Conference of the North American Chapter of the Association for Computational Linguistics: Human Language Technologies. 4171-4186.

[4] Markus Diem, Florian Kleber, and Robert Sablatnig. 2011. Text Classification and Document Layout Analysis of Paper Fragments. In 2011 International Conference on Document Analysis and Recognition. 854-858.

[5] Zhangyin Feng, Daya Guo, Duyu Tang, Nan Duan, Xiaocheng Feng, Ming Gong, Linjun Shou, Bing Qin, Ting Liu, Daxin Jiang, and Ming Zhou. 2020. CodeBERT: A Pre-Trained Model for Programming and Natural Languages. In Findings of the Association for Computational Linguistics: EMNLP 2020. 1536-1547.

[6] Kaiming He, X. Zhang, Shaoqing Ren, and Jian Sun. 2016. Deep Residual Learning for Image Recognition. IEEE Conference on Computer Vision and Pattern Recognition (2016), 770-778.

[7] Akash Abdu Jyothi, Thibaut Durand, Jiawei He, Leonid Sigal, and Greg Mori. 2019 LayoutVAE: Stochastic Scene Layout Generation from a Label Set. In International Conference on Computer Vision.

[8] Guolin Ke, Qi Meng, Thomas Finely, Taifeng Wang, Wei Chen, Weidong Ma, Qiwei Ye, and Tie-Yan Liu. 2017. LightGBM: A Highly Efficient Gradient Boosting Decision Tree. In Advances in Neural Information Processing Systems.

[9] Diederik P. Kingma and Jimmy Ba. 2015. Adam: A Method for Stochastic Optimization. In 3rd International Conference on Learning Representations, Yoshua Bengio and Yann LeCun (Eds.)

[10] Hsin-Ying Lee, Weilong Yang, Lu Jiang, Madison Le, Irfan Essa, Haifeng Gong, and Ming-Hsuan Yang. 2020. Neural Design Network: Graphic Layout Generation with Constraints. In Proceedings of European Conference on Computer Vision.

[11] Jianan Li, Jimei Yang, Aaron Hertzmann, Jianming Zhang, and Tingfa Xu. 2019 LayoutGAN: Generating Graphic Layouts with Wireframe Discriminators.

[12] Jianan Li, Jimei Yang, Aaron Hertzmann, Jianming Zhang, and Tingfa Xu. 2019. LayoutGAN: Generating Graphic Layouts with Wireframe Discriminators. In 7th International Conference on Learning Representations.

[13] Yang Li, J. Amelot, X. Zhou, S. Bengio, and S. Si. 2020. Auto Completion of User Interface Layout Design Using Transformer-Based Tree Decoders. abs/2001.05308 (2020).

[14] Tsung-Yi Lin, Priya Goyal, Ross Girshick, Kaiming He, and Piotr Dollar. 2017. Focal Loss for Dense Object Detection. 2017 IEEE International Conference on Computer Vision (2017).

[15] Simon Lok, Steven Feiner, and Gary Ngai. 2004. Evaluation of Visual Balance for Automated Layout. Association for Computing Machinery, 101-108.

[16] Jiasen Lu, Dhruv Batra, Devi Parikh, and Stefan Lee. 2019. ViLBERT: Pretraining Task-Agnostic Visiolinguistic Representations for Vision-and-Language Tasks. In Advances in Neural Information Processing Systems 32: Annual Conference on Neural Information Processing Systems 2019, NeurIPS 2019, 8-14 December 2019, Vancouver, BC, Canada, Hanna M. Wallach, Hugo Larochelle, Alina Beygelzimer, Florence d'Alché-Buc, Emily B. Fox, and Roman Garnett (Eds.). 13-23.

[17] Razvan Pascanu, Tomas Mikolov, and Yoshua Bengio. 2012. On the difficulty of training Recurrent Neural Networks.
[18] A. Patil, Omri Ben-Eliezer, Or Perel, and Hadar Averbuch-Elor. 2020. READ: Recursive Autoencoders for Document Layout Generation. 2020 IEEE/CVF Conference on Computer Vision and Pattern Recognition Workshops (2020), 2316-2325.

[19] Matthew E. Peters, Mark Neumann, Mohit Iyyer, Matt Gardner, Christopher Clark, Kenton Lee, and Luke Zettlemoyer. 2018. Deep contextualized word representations. In Proceedings of the 2019 Conference of the North American Chapter of the Association for Computational Linguistics: Human Language Technologies. 2227-2237.

[20] A. Radford. 2018. Improving Language Understanding by Generative PreTraining.

[21] Justus J. Randolph. 2005. Free-marginal multirater kappa (multirater k [free]): An alternative to fleiss' fixed-marginal multirater kapp. (2005).

[22] Shaoqing Ren, Kaiming He, Ross Girshick, and Jian Sun. 2015. Faster R-CNN: Towards Real-Time Object Detection with Region Proposal Networks. In Advances in Neural Information Processing Systems, Vol. 28. 91-99.

[23] Karen Simonyan and Andrew Zisserman. 2015. Very Deep Convolutional Networks for Large-Scale Image Recognition. In International Conference on Learning Representations.

[24] Carlos Soto and Shinjae Yoo. 2019. Visual Detection with Context for Document Layout Analysis. In Proceedings of the 2019 Conference on Empirical Methods in Natural Language Processing and the 9th International foint Conference on Natural Language Processing. 3464-3470.

[25] A. Stoffel, David Spretke, Henrik Kinnemann, and D. Keim. 2010. Enhancing document structure analysis using visual analytics. In Proceedings of the 2010 ACM Symposium on Applied Computing.

[26] Chen Sun, Austin Myers, Carl Vondrick, Kevin Murphy, and Cordelia Schmid. 2019. VideoBERT: A Joint Model for Video and Language Representation Learning. 2019 IEEE/CVF International Conference on Computer Vision (2019).

[27] S. Tabata, Hiroki Yoshihara, H. Maeda, and Kei Yokoyama. 2019. Automatic layout generation for graphical design magazines. ACM SIGGRAPH 2019 Posters (2019).

[28] Kai Sheng Tai, Richard Socher, and Christopher D. Manning. 2015. Improved Semantic Representations From Tree-Structured Long Short-Term Memory Networks. In Proceedings of the 53rd Annual Meeting of the Association for Computational Linguistics and the 7th International foint Conference on Natural Language Processing.

[29] Ashish Vaswani, Noam Shazeer, Niki Parmar, Jakob Uszkoreit, Llion Jones, Aidan N. Gomez, Lukasz Kaiser, and Illia Polosukhin. 2017. Attention Is All You Need.

[30] Wenhui Wang, Nan Yang, Furu Wei, Baobao Chang, and Ming Zhou. 2017. Gated Self-Matching Networks for Reading Comprehension and Question Answering. In Proceedings of the 55th Annual Meeting of the Association for Computational Linguistics. Association for Computational Linguistics, 189-198.

[31] Zhiyong Wu, Yun Chen, Ben Kao, and Qun Liu. 2020. Perturbed Masking: Parameter-free Probing for Analyzing and Interpreting BERT. Proceedings of the 58th Annual Meeting of the Association for Computational Linguistics (2020).

[32] Yiheng Xu, Minghao Li, Lei Cui, Shaohan Huang, Furu Wei, and Ming Zhou. 2019. LayoutLM: Pre-training of Text and Layout for Document Image Understanding.

[33] Zhilin Yang, Zihang Dai, Yiming Yang, Jaime G. Carbonell, Ruslan Salakhutdinov, and Quoc V. Le. 2019. XLNet: Generalized Autoregressive Pretraining for Language Understanding. In Advances in Neural Information Processing Systems 32: Annual Conference on Neural Information Processing Systems 2019, NeurIPS 2019, 8-14 December 2019, Vancouver, BC, Canada, Hanna M. Wallach, Hugo Larochelle, Alina Beygelzimer, Florence d'Alché-Buc, Emily B. Fox, and Roman Garnett (Eds.). 5754-5764.

[34] Xinru Zheng, Xiaotian Qiao, Ying Cao, and Rynson W.H. Lau. 2019. Contentaware Generative Modeling of Graphic Design Layouts. ACM Transactions on Graphics (2019). 